\title{
Streptococcus equi subspecies zooepidemicus Meningitis, Septicemia, and Brain Infarcts in a Costa Rican Infant
}

Fabricio Sevilla-Acosta ${ }^{1,2}$, Angela Ballestero-Pernudi ${ }^{2}$, Elisandro Jiménez-Cruz ${ }^{3}$, Hazel ÁlvarezCabalceta $^{3}$, Gabriela Naranjo-Zuñiga ${ }^{4}$

1. Pediatrics, Hospital Nacional De Niños "Dr. Carlos Sáenz Herrera", San José, CRI 2. Pediatrics, Hospital La Anexión, Nicoya, CRI 3. Microbiology, Hospital La Anexión, Nicoya, CRI 4. Infectious Disease, Hospital Nacional De Niños "Dr. Carlos Sáenz Herrera", San José, CRI

Corresponding author: Fabricio Sevilla-Acosta, fsevilla01@msn.com

\begin{abstract}
Streptococcus equi, an equine commensal bacterium, is a rare etiology of septicemia and meningoencephalitis in humans and is extremely infrequent in children. Scarce literature has been published about its clinical presentation, treatment and outcomes in infants. Here, we describe a case of $S$. equi subsp. zooepidemicus septicemia and meningoencephalitis in a five-month-old Costa Rican infant that was confirmed by peripheral blood and cerebrospinal fluid (CSF) cultures in a regional hospital of the Pacific coast of Costa Rica who developed multiple ischemic cerebral infarcts secondary to infectious vasculitis, a subdural empyema and serious neurological sequelae. We also reviewed the literature on S. equi meningitis in infants under one year of age. This is the first reported case in our country, the fourth reported in infants under one year of age and the second describing multiple cerebral infarcts and subdural empyema in an infant.
\end{abstract}

Categories: Neurology, Pediatrics, Infectious Disease

Keywords: streptococcus equi, septic shock in children, gram-positive meningitis, infants, brain infarcts, subdural empyema

\section{Introduction}

Streptococcus equi is a beta-hemolytic group C Streptococcus. There are four Streptococcus in the Lancefield group C: S. equi subsp. equi, S. equi subsp. zooepidemicus, S. dysgalactiae subsp. dysgalactiae, and S. dysgalactiae subsp. equisimilis [1]. All of them can cause disease in humans but are considered rare diseases. S. equi subsp. zooepidemicus is a zoonotic and opportunistic pathogen closely related to $S$. equi subsp. equi; both share virulence factors with Streptococcus pyogenes and can cause severe disease in humans [2].

Review began $07 / 31 / 2021$ Review ended 08/12/2021 Published 08/18/2021

\section{(๑) Copyright 2021}

Sevilla-Acosta et al. This is an open access article distributed under the terms of the Creative Commons Attribution License CC-BY 4.0., which permits unrestricted use, distribution, and reproduction in any medium, provided the original author and source are credited.
S. equi subsp. zooepidemicus often colonizes and causes disease in horses, but has also been described to cause disease in other mammals (swine, cattle, guinea pigs and sheep). In humans it is an uncommon zoonotic disease with very few reported cases. Infections reported in humans range from mild diseases as pharyngitis, skin and soft tissue infections to severe diseases as epiglottitis, pneumonitis, septic arthritis, osteomyelitis, peritonitis and even bacteremia; endocarditis and meningitis have also been described [1-3]. The source of the infection is associated with direct horse contact, ingestion of unpasteurized dairy products or contact with infected mammals' secretions [1-3]. Most of the infections previously reported occurred in adult patients. $S$. equi infections in children are very rare and it is presumed that children who are immunologically compromised would be more susceptible to infection with this pathogen [1].

World literature describes approximately 50 cases of group C streptococci meningitis, most of them in adult patients [1]. S. equi subsp. zooepidemicus appears to be the most common subspecies of group C Streptococcus to cause meningitis [4]. In the literature, we found only three isolated published cases of children under one year of age with $S$. equi subsp. zooepidemicus meningitis $[3,5,6]$. It is considered a rare disease in all age groups and is associated with poor outcomes [7]. To our knowledge, no other author has analyzed the burden of this disease in children, especially in infants under one year of age.

We describe the case of a healthy Costa Rican infant with septicemia and meningoencephalitis caused by $S$. equi subsp. zooepidemicus confirmed by both peripheral blood and cerebrospinal fluid (CSF) cultures, who developed multiple ischemic cerebral infarcts secondary to infectious vasculitis, a subdural empyema and serious neurological sequelae. This is the first reported case in our country, the fourth reported in infancy in the literature and the second requiring neurosurgical drainage of a brain subdural empyema and describing multiple cerebral infarcts in an infant.

\section{Case Presentation}

A five-month-old male was admitted to La Anexión Hospital, a community hospital on the Pacific coast of 


\section{Cureus}

Costa Rica, with a history of febrile status epilepticus. He had experienced four days of fever and vomiting. On admission, physical examination revealed a febrile encephalopathic infant with obvious signs of intracranial hypertension (altered mental status, setting-sun sign, bulging fontanel, arterial hypertension and bradycardia) associated with focal seizures and septic shock.

He needed immediate endotracheal intubation, mechanical ventilation, intravenous (IV) fluids and antiepileptic drugs (diazepam and phenytoin). Peripheral blood cultures and blood samples were obtained for laboratory analysis. Meningeal doses of intravenous cefotaxime were started as empiric therapy and lumbar puncture was deferred at that moment because of intracranial hypertension.

Complete blood count revealed discrete anemia and leukocytosis with normal platelets (hemoglobin: 10.1 g/dL; leukocytes: 14,490/mm3; platelets: 198,000/mm3). Coagulation profile was slightly abnormal (prothrombin time [PT]: 51\%, activated partial thromboplastin time [APTT]: 20s, international normalised ratio [INR]: 1.46). C-reactive protein (CRP) was negative $(6 \mathrm{mg} / \mathrm{L})$ and procalcitonin (PCT) was positive $(21$ $\mathrm{ng} / \mathrm{mL}$ ). Creatinine, blood urea nitrogen, electrolytes and liver enzymes were normal. Respiratory panel was negative including severe acute respiratory syndrome coronavirus 2 (SARS-CoV-2) and brain ultrasound revealed hydrocephalus, without echogenic interior material and no extra-axial collections.

Patient was transferred to the pediatric intensive care unit (PICU) of the Hospital Nacional de Niños "Dr. Carlos Sáenz Herrera", the only national tertiary pediatric center in our country. Brain CT scan showed multifocal hypodense lesions localized in basal ganglia and cerebral cortex (Figure 1). Brain MRI showed T1 hypointense and T2-FLAIR hyperintense lesions localized in bilateral temporal lobes, right frontal lobe, left occipital lobe and basal ganglia (thalamus, caudate nucleus, putamen and globus pallidus). These lesions were apparent diffusion coefficient (ADC) hypointense and diffusion-weighted imaging (DWI) hyperintense, confirming ischemic events secondary to infectious vasculitis.

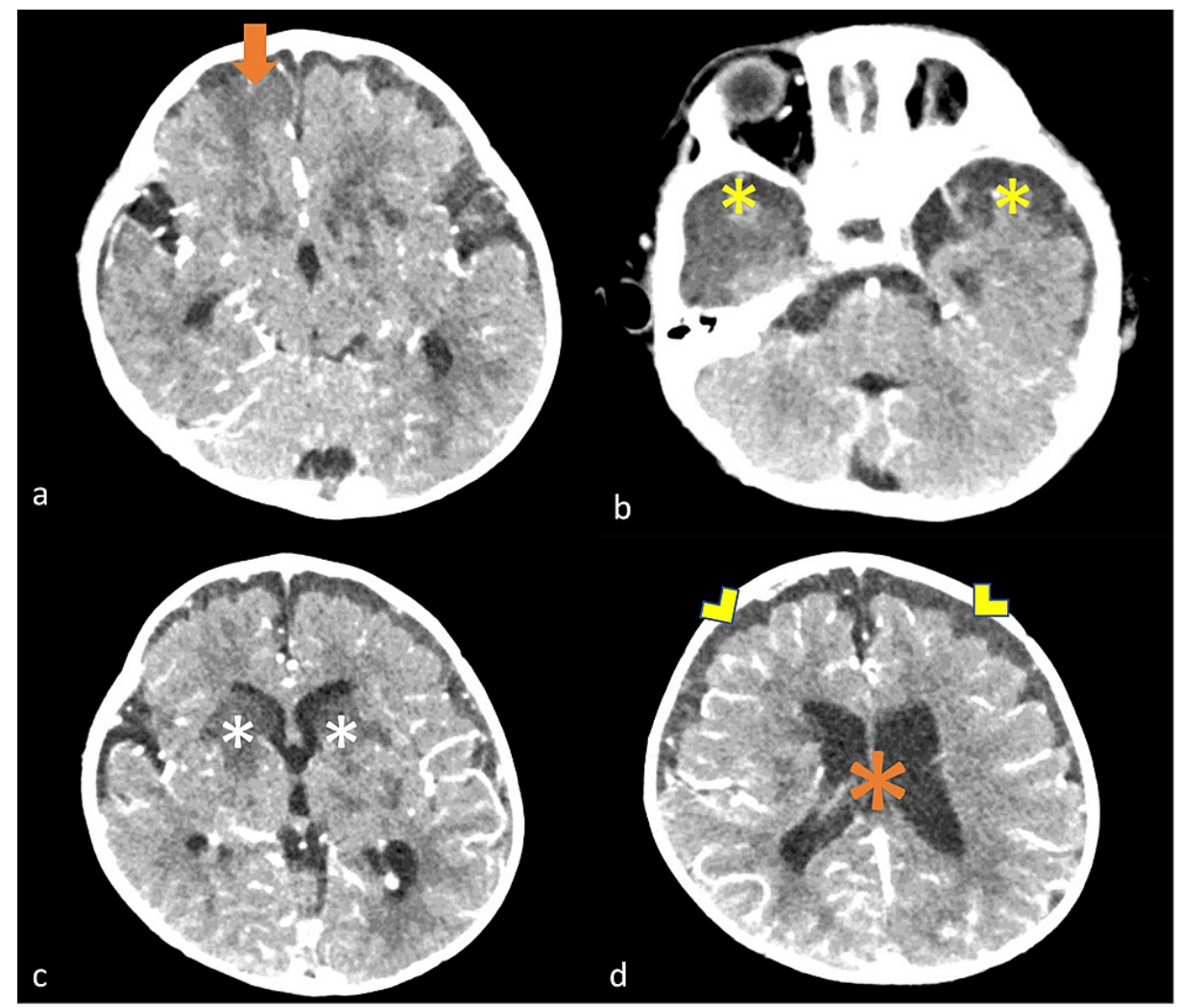

FIGURE 1: Brain CT scan at admission to the pediatric intensive care unit.

a) Hypodense lesions in the right frontal lobe (orange arrow); b) Bilateral temporal lobe hypodense lesions (yellow asterisk); c) Bilateral basal ganglia hypodense lesions (white asterisks); d) Ventriculomegaly (orange asterisk) and early subdural empyema (yellow arrowheads).

At the PICU, CSF analysis was obtained and revealed hipoglucorraquia (undetectable glucose), elevated proteins (256 mg/dL), pleocytosis (leucocytes: 265/mm3; 50\% lymphocytes; 50\% neutrophils) and Grampositive bacteria in chains on Gram stain. Biofire FilmArray (bioMérieux, Marcy L`Etoile, France) Meningitis/Encephalitis panel was negative for most common pathogens. 
On the second PICU day, blood cultures taken at the referral hospital and CSF taken at the PICU grew large colonies of Lancefield group C beta-hemolytic Streptococcus. Further characterization identified the organism as Streptococcus equi subsp. zooepidemicus, both in blood smears and CSF (Figure 2). Blood cultures were taken on admission from two different sites and inoculated in a blood agar plate at a temperature of $37^{\circ} \mathrm{C}$. Multiple colonies of Gram-positive cocci in chains were seen at the microscope. At 24h of inoculation, there was growing of multiple colonies of streptococci, of transparent aspect with a $1 \mathrm{~cm}$ diameter of betahemolysis. We identified the germ using VITEK 2 (bioMérieux), which detected Streptococcus equi subsp. zooepidemicus with a probability of $93 \%$ in both blood cultures. Sensitivity testing was done using AST-ST03 card and determined that the organism was sensitive to beta-lactams (minimum inhibitory concentrations [MIC]: ampicillin $=0.25 \mu \mathrm{g} / \mathrm{ml}$; penicillin $=0.06 \mu \mathrm{g} / \mathrm{ml}$; cefotaxime $=0.12 \mu \mathrm{g} / \mathrm{ml}$; ceftriaxone $=0.12 \mu \mathrm{g} / \mathrm{ml}$ ), vancomycin (MIC: $0.25 \mu \mathrm{g} / \mathrm{ml}$ ), chloramphenicol (MIC: $4 \mu \mathrm{g} / \mathrm{ml}$ ), linezolid (MIC: $2 \mu \mathrm{g} / \mathrm{ml}$ ), rifampicin (MIC: 0.06 ), intermediate to quinolones (MIC: levofloxacin $=2 \mu \mathrm{g} / \mathrm{ml}$; moxifloxacin $=0.5 \mu \mathrm{g} / \mathrm{ml}$ ) and resistant only to trimethoprim/sulfamethoxazole (MIC: $160 \mathrm{\mu g} / \mathrm{ml}$ ). Finally, the result of the blood cultures was confirmed when the same agent was isolated from the CSF at $24 \mathrm{~h}$ of inoculation.

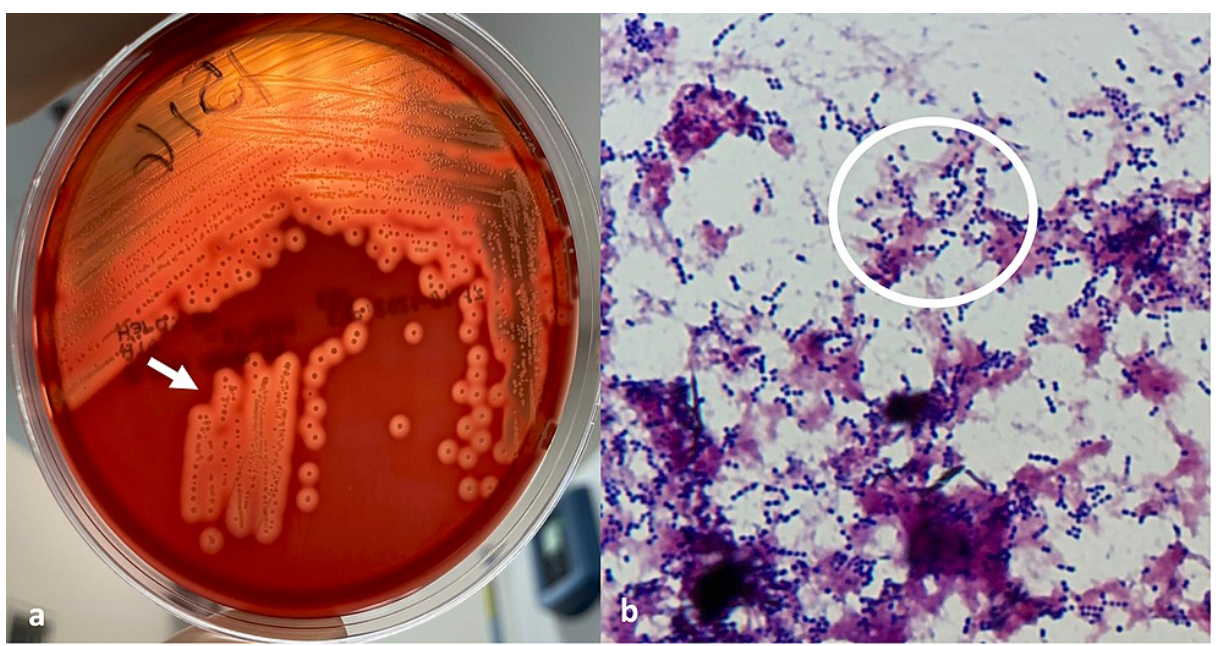

\section{FIGURE 2: Cultures of peripheral blood.}

a) Beta-hemolytic large colony of Lancefield group C streptococcus in blood agar (white arrow); b) Growing of multiple Gram-positive streptococci in Gram stain (white circle).

On the seventh day of PICU stay, patient had neurological deterioration and a new brain CT scan revealed a subdural collection for which a subdural drainage was needed. He completed a total of 10 days of mechanical ventilation, 19 days of cefotaxime, 14 days of vancomycin and seven days of amikacin.

Neurological examination prior to discharge was consistent with serious neurological compromise: axial hypotonus and spastic quadriplegia. Electroencephalogram (EEG) was abnormal with poor organization and slow basal activity but without paroxysms. Hearing screen revealed severe bilateral sensorineural hearing loss. Patient was discharged feeding across nasogastric tube and an intensive physical therapy program was started.

Epidemiological investigation didn't determine any contact with horses or consumption of unpasteurized milk, but depriving socio-cultural status and unhygienic conditions were seen in their home visit. There was a similar case of a 30-year-old adult in the same geographic area 14 days prior to our patient admission who presented encephalopathic to La Anexión Hospital, and Streptococcus equi subsp. zooepidemicus was isolated from CSF as well. He had negative blood cultures and fully recovered after 10 days of IV vancomycin. He required three days of mechanical ventilation and brain CT scan was normal. A common origin couldn't be determined between these two cases.

Currently, the patient is one year old and has spastic quadriplegia, severe bilateral hearing loss and feeds by gastrostomy tube. Antiepileptic drugs are still needed (carbamazepine) because he had another status epilepticus after discharge and receives intensive physical therapy weekly.

\section{Discussion}

This is the first case of an infant affected by Streptococcus equi subsp. zooepidemicus reported in our country. Bloodstream infections and meningoencephalitis in children in Costa Rica are mainly due to Staphylococcus aureus and Streptococcus pneumoniae. In this particular age group of our patient, Streptococcus agalactiae is the leading cause. Especially for this patient, Streptococcus agalactiae was highly suspected at the beginning. Blood cultures documented multiple colonies of Gram-positive streptococci with beta-hemolysis, so empiric 
antibiotics were targeted to the most frequent streptococci in our environment at meningeal doses. Of note, the result of the Biofire FilmArray (bioMérieux) Meningitis/Encephalitis panel made in the CSF was negative, suggesting that the causative agent was not a common Streptococcus. Finally, the results of the blood cultures identified Streptococcus equi subsp. zooepidemicus and were confirmed when the same agent was isolated from the CSF.

As reported in other cases in humans, Streptococcus equi infections have mostly been reported with the subspecies zooepidemicus. Clinical scenarios have been: unknown focus bacteremia, meningitis, endocarditis, and there are also cases of aortitis. These case reports found that consumption of unpasteurized milk products and contact with horses or secretions infected with this organism can be determined in the majority of cases and that the fatality rate of these infections has been especially high in humans [8]. In our patient, there was no identifiable contact with animals or with unpasteurized milk products; apparently this baby received only breastfeeding. However, depriving socio-cultural status and unhygienic conditions were seen in their home visit. Costa Rica is a tropical country and especially the Pacific coast of Guanacaste is an area with a lot of livestock activity, so it is possible that somewhere in the patient's house he could have contact with secretions of this kind of animals or domestic animals, as it is also known that even dogs can transmit the illness [3]. Of especial interest, there was a report of an adult patient living in the same geographic area who developed Streptococcus equi subsp. zooepidemicus meningitis in the previous 14 days, suggesting a common origin of both cases, however, this association couldn't be determined by the epidemiological investigation. It seems that this infection is more severe in children than in adults, as the adult patient fully recovered and our patient had severe neurological sequelae. Probably, the infant developed this catastrophic course of the diseases because of his immature immunological system and a high burden of the pathogen, as demonstrated by the isolation of the pathogen both in blood and CSF.

Other authors have previously performed a literature search and review of Streptococcus equi meningitis. van Samkar et al. in 2016 [7] and Torpiano et al. in 2020 [9] made a literature search in PubMed using the terms "Streptococcus equi" and "meningitis". We actualized this data with the cases published in PubMed since then and our two cases, with especial interest in the reported cases of infants under one year of age. Using the same words in a literature search in PubMed, actually there are 37 reported cases of $S$. equi meningitis caused by both subspecies between 1978 and 2021, 32 of which were caused by subspecies zooepidemicus, and three caused by subspecies equi (two cases were reported as $S$. equi meningitis but did not distinguish between subspecies) [1-18]. S. equi meningitis in children is especially infrequent as the majority of cases reported have been in adult patients (>70 years: 14 patients; $40-69$ years: 10 patients; 20 -39 years: six patients; 10-19 years: three patients; 1-9 years: 0 patients), and only four cases were under one year of age $[3,5,6,18]$. Here we report two more cases: a five-month-old infant and a thirty-year-old male. Combining these data with our two cases, there are now 39 reported $S$. equi meningitis cases, in which seven cases occurred in the pediatric age and five were under one year of age.

There are only six previous pediatric meningitis cases by $S$. equi reported in the literature (see Table 1), including a one-day-old male who died [5], a one-day-old male who recovered completely [18], a 14-weekold male who also recovered completely [18], a six-month-old female who had severe hearing loss and mild neurocognitive deficits [3], a 13-year-old female who had unilateral hearing defect [10] and a 13-year-old male who recovered well without sequelae [9]. 


\begin{tabular}{|c|c|c|c|c|c|c|c|c|}
\hline \# & $\begin{array}{l}\text { Country, } \\
\text { year }\end{array}$ & Age & Sex & Subspecies & Disease features & Treatment (Duration) & Outcome & Reference \\
\hline 1 & $\begin{array}{l}\text { UK, } \\
1984\end{array}$ & 1 day & Male & Zooepidemicus & Septicemia, Meningitis & Not specified & Died & [5] \\
\hline 2 & $\begin{array}{l}\text { USA, } \\
1997\end{array}$ & 1 day & Male & Not specified & Meningitis & Ampicillin (10 days) & $\begin{array}{l}\text { Recovered } \\
\text { completely }\end{array}$ & [18] \\
\hline 3 & $\begin{array}{l}\text { UK, } \\
2000\end{array}$ & $\begin{array}{l}14 \\
\text { weeks }\end{array}$ & Male & Zooepidemicus & Septicemia, Meningitis & Ceftriaxone ( 3 weeks) & $\begin{array}{l}\text { Recovered } \\
\text { completely }\end{array}$ & [18] \\
\hline 4 & $\begin{array}{l}\text { CR, } \\
2021\end{array}$ & $\begin{array}{l}5 \\
\text { months }\end{array}$ & Male & Zooepidemicus & $\begin{array}{l}\text { Septicemia, } \\
\text { Meningitis, Multifocal brain } \\
\text { infarctions, Subdural } \\
\text { empyema }\end{array}$ & $\begin{array}{l}\text { Cetotaxime (19 days), } \\
\text { Vancomycin (14 days), Amikacin (7 } \\
\text { days) }\end{array}$ & $\begin{array}{l}\text { Cerebral palsy, } \\
\text { severe bilateral } \\
\text { hearing loss, epilepsy }\end{array}$ & [*] \\
\hline 5 & $\begin{array}{l}\text { USA, } \\
2019\end{array}$ & $\begin{array}{l}6 \\
\text { months }\end{array}$ & Female & Zooepidemicus & $\begin{array}{l}\text { Septicemia, Meningitis, } \\
\text { Multifocal brain infarctions, } \\
\text { Subdural empyema }\end{array}$ & $\begin{array}{l}\text { Ceftriaxone ( } 2 \text { days), Vancomycin ( } 2 \\
\text { days), Dexamethasone ( } 2 \text { days), } \\
\text { Penicillin G ( } 4 \text { weeks) }\end{array}$ & $\begin{array}{l}\text { Severe hearing loss } \\
\text { and mild } \\
\text { neurocognitive } \\
\text { deficits }\end{array}$ & [3] \\
\hline 6 & $\begin{array}{l}\text { USA, } \\
2001\end{array}$ & $\begin{array}{l}13 \\
\text { years }\end{array}$ & Female & Zooepidemicus & Septicemia, Meningitis & Ceftriaxone + Vancomycin (14 days) & $\begin{array}{l}\text { Unilateral hearing } \\
\text { defect }\end{array}$ & [10] \\
\hline 7 & $\begin{array}{l}\text { Malta, } \\
2020\end{array}$ & $\begin{array}{l}13 \\
\text { years }\end{array}$ & Male & Equi & $\begin{array}{l}\text { Septicemia, Meningitis, } \\
\text { Subdural empyema }\end{array}$ & Ceftriaxone + Rifampicin (8 weeks) & $\begin{array}{l}\text { Recovered } \\
\text { completely }\end{array}$ & [9] \\
\hline
\end{tabular}

TABLE 1: Streptococcus equi meningitis in pediatric patients (sorted by age).

CR: Costa Rica; UK: United Kingdom; USA: United States of America. *Current reported case.

Our patient is the fifth case of $S$. equi meningitis reported in an infant under one year of age, all of them caused by the subspecies zooepidemicus, except one case in which authors did not distinguish between subspecies (Table 1). One patient died (20\%), of the surviving four patients, two had neurological sequelae (50\%) and two recovered completely (50\%). The most common sequela was hearing loss and, unfortunately, our case is the first to report cerebral palsy and epilepsy as permanent neurological sequelae. In this age group, the mortality rate seems to be the same as previously reported in other age groups (20\%) but permanent neurological morbidity seems to be higher than reported in adults ( $50 \%$ in infants under one year versus $31.4 \%$ in adults) [9].

In previous reviews of $S$. equi meningitis, cranial CT scan showed abnormalities in $50 \%$ of patients (brain edema, sinusitis, mastoiditis, brain abscesses, hydrocephalus and cerebral hypodensities were the more common abnormalities), and only two out of 33 patients older than one year developed a subdural empyema (6\%), in contrast with infants under one year of age, in which two out of five required neurosurgical drainage of subdural empyema (40\%) [7,9]. As bacteremia is more frequent in infants under one year, this could explain the higher incidence of intracranial and subdural complications in this age group. It is known in mouse models of infection that some virulence factors of $S$. equi subsp. zooepidemicus induce meningitis by breaking through the blood-brain barrier (BBB). High bacteremia is necessary for binding to the brain microvascular endothelium of the BBB and inducing meningitis [19]. In our patient multiple ischemic cerebral infarcts secondary to infectious vasculitis were also documented, an intracranial complication reported only in one previous patient. In this age group the brain insult is determinant because an injury to the developing brain can lead to cerebral palsy. As an example, Torpiano et al. reported a case of a 13-yearold male who was complicated by a subdural empyema secondary to $S$. equi subsp. equi meningitis and fully recovered after neurosurgical drainage and antibiotic treatment [9]. Although this case was due to $S$. equi subsp. equi, and our case was due to $S$. equi subsp. zooepidemicus, both were complicated with subdural empyema and required neurosurgical evacuation, but it seems that in our case the worst outcome could be determined because of the age of the child and the associated intracranial complications.

Empirical antibiotic therapy for pyogenic meningitis includes a third-generation cephalosporin, vancomicin and steroids instituted before the first dose of antibiotics until pneumococcus is ruled out [14]. Once the causative microorganism is identified, treatment should be guided by culture sensitivity report. The recommended antimicrobial therapy for streptococcal meningitis caused by an equine pathogen is penicillin [7]. Bactericidal activity against group $\mathrm{C}$ streptococci is achieved when adding gentamicin or rifampin to a $\beta$-lactam or vancomycin [14]. There are no published data to recommend routine use of corticosteroids in meningitis caused by $S$. equi. In our case the patient received 19 days of cefotaxime, 14 days of vancomycin 
and seven days of amikacin as adjunctive therapy because of the complication of the subdural empyema. In the literature the duration of antibiotic therapy ranged between 10 days to eight weeks (Table 1)

$[3,6,9,10,13,14,18]$.

\section{Conclusions}

We describe the case of a five-month-old male presenting with meningoencephalitis and septicemia by $S$. equi subsp. zooepidemicus who developed multiple ischemic cerebral infarcts secondary to infectious vasculitis, a subdural empyema and serious permanent neurological sequelae. This case warns of a potentially devastating infection by an invasive equine pathogen that should be promptly treated with penicillin or cephalosporin and in the presence of subdural collections may require surgical drainage. Despite rapid institution of appropriate treatment, this infection could lead to death in $20 \%$ of patients and severe neurological sequelae in $50 \%$ of infants under one year of age, including cerebral palsy, so prevention is the cornerstone of treatment. Prevention of this disease includes avoiding contact with horses and avoiding consumption of unpasteurized cow's milk. This case report and the review of the literature reminds us that, although infrequent, meningoencephalitis by $S$. equi is a serious threat to human health and could lead to permanent neurological sequelae in children, especially in infants under one year of age.

\section{Additional Information \\ Disclosures}

Human subjects: Consent was obtained or waived by all participants in this study. Conflicts of interest: In compliance with the ICMJE uniform disclosure form, all authors declare the following: Payment/services info: All authors have declared that no financial support was received from any organization for the submitted work. Financial relationships: All authors have declared that they have no financial relationships at present or within the previous three years with any organizations that might have an interest in the submitted work. Other relationships: All authors have declared that there are no other relationships or activities that could appear to have influenced the submitted work.

\section{References}

1. Elsayed S, Hammerberg O, Massey V, Hussain Z: Streptococcus equi subspecies equi (Lancefield group C) meningitis in a child. Clin Microbiol Infect. 2003, 9:869-72. 10.1046/j.1469-0691.2003.00663.x

2. Pelkonen S, Lindahl SB, Suomala P, et al.: Transmission of Streptococcus equi subspecies zooepidemicus infection from horses to humans. Emerg Infect Dis. 2013, 19:1041-8. 10.3201/eid1907.121365

3. Zahlanie Y, Almatrafi M, Filkins L, Hsiang MS: Possible canine source of Streptococcus equi subspecies zooepidemicus causing meningitis in an infant. IDCases. 2019, 31:e00568. 10.1016/j.idcr.2019.e00568

4. Minces LR, Brown PJ, Veldkamp PJ: Human meningitis from Streptococcus equi subsp. zooepidemicus acquired as zoonoses. Epidemiol Infect. 2011, 139:406-10. 10.1017/S0950268810001184

5. Edwards AT, Roulson M, Ironside MJ: A milk-borne outbreak of serious infection due to Streptococcus zooepidemicus (Lancefield Group C). Epidemiol Infect. 1988, 101:43-51. 10.1017/s0950268800029204

6. Rajasekhar A, Clancy CJ: Meningitis due to group C Streptococcus: a case report and review of the literature Scand J Infect Dis. 2010, 42:571-8. 10.3109/00365541003754428

7. van Samkar A, Brouwer MC, van der Ende A, van de Beek D: Streptococcus equi meningitis. Clin Microbiol Infect. 2016, 22:e3-4. 10.1016/j.cmi.2015.09.003

8. Trell K, Nilson B, Petersson AC, Rasmussen M: Clinical and microbiological features of bacteremia with Streptococcus equi. Diagn Microbiol Infect Dis. 2017, 87:196-8. 10.1016/j.diagmicrobio.2016.10.018

9. Torpiano P, Nestorova N, Vella C: Streptococcus equi subsp. equi meningitis, septicemia and subdural empyema in a child. IDCases. 2020, 14:e00808. 10.1016/j.idcr.2020.e00808

10. Shah SS, Matthews RP, Cohen C: Group C streptococcal meningitis: case report and review of the literature. Pediatr Infect Dis J. 2001, 20:445-8. 10.1097/00006454-200104000-00016

11. Popescu GA, Fuerea R, Benea E: Meningitis due to an unusual human pathogen: Streptococcus equi subspecies equi. South Med J. 2006, 99:190-1. 10.1097/01.smj.0000198265.82867.a3

12. Jovanović M, Stevanović G, Tošić T, Stošović B, Zervos MJ: Streptococcus equi subsp. zooepidemicus meningitis. J Med Microbiol. 2008, 57:373-5. 10.1099/jmm.0.47487-0

13. Eyre DW, Kenkre JS, Bowler IC, McBride SJ: Streptococcus equi subspecies zooepidemicus meningitis--a case report and review of the literature. Eur J Clin Microbiol Infect Dis. 2010, 29:1459-63. 10.1007/s 10096010-1037-5

14. Aida Z, Lamia A, Souheil Z, et al.: Meningitis due to Streptococcus equi in a 73 year old woman with an osteodural defect. IDCases. 2020, 21:e00779. 10.1016/j.idcr.2020.e00779

15. Madžar D, Hagge M, Möller S, Regensburger M, Lee DH, Schwab S, Jantsch J: Endogenous endophthalmitis complicating Streptococcus equi subspecies zooepidemicus meningitis: a case report. BMC Res Notes. 2015, 8:184. 10.1186/s13104-015-1133-9

16. Mori N, Guevara JM, Tilley DH, Briceno JA, Zunt JR, Montano SM: Streptococcus equi subsp. zooepidemicus meningitis in Peru. J Med Microbiol. 2013, 62:335-7. 10.1099/jmm.0.050245-0

17. Poulin MF, Boivin G: A case of disseminated infection caused by Streptococcus equi subspecies zooepidemicus. Can J Infect Dis Med Microbiol. 2009, 20:59-61. 10.1155/2009/538967

18. Jenkins EL, McGuire W: Group C streptococcal meningitis in infancy. Acta Paediatr. 2000, 89:1141-2. 10.1080/713794563

19. Zhe M, Jie P, Hui Z, et al.: SILAC and LC-MS/MS identification of Streptococcus equi ssp. zooepidemicus proteins that contribute to mouse brain microvascular endothelial cell infection. Appl Microbiol Biotechnol. 2016, 100:7125-36. 10.1007/s00253-016-7579-4 